\title{
Niveles aumentados de estrés oxidativo se asocian a disfunción endotelial periférica y respuesta vascular pulmonar disminuida frente a vasodilatadores en pacientes con hipertensión pulmonar.
} \author{
Lorena García?. \\ 1 Pontificia Universidad Católica de Chile. Departamento de \\ Enfermedades Cardiovasculares \\ 2 Centro FONDAP, Estudios moleculares de la célula, Instituto de Ciencias \\ Biomédicas Facultad de Medicina. Universidad de Chile.
}

Luigi Gabrielli', Pablo Castro', Mario Chiong², Hernán Alcaíno², Hugo Verdejo', Mario Navarro Douglas Greig', Iván Godoy ${ }^{1}$, Barbra Toro², Clara Quiroga2, Guillermo Díaz-Araya², Sergio Lavandero²,

\section{Resumen:}

Introducción: La Hipertensión arterial pulmonar (HP) se caracteriza por remodelado vascular y disfunción endotelial. Evidencia experimental muestra que el estrés oxidativo juega un rol importante en la patogénesis de la HP. El rol del estrés oxidativo, su relación con la función endotelial periférica y con la respuesta vascular pulmonar a vasodilatadores en pacientes con HP no está aclarada. Objetivo: evaluar parámetros de estrés oxidativo y función endotelial periférica en pacientes con HP y estudiar su relación con la respuesta vascular pulmonar frente a vasodilatadores.

Métodos: estudio transversal. Se incluyeron 14 pacientes con HP y 14 controles pareados por edad y sexo. En todos los sujetos se midieron: niveles plasmáticos de malondialdehído (MDA), superóxido dismutasa ligada a endotelio (eSOD) y xantino oxidasa (eXO). Vasodilatación dependiente de endotelio mediada por flujo en arteria braquial fue usada como marcador de función endotelial (FDD). Función ventricular derecha y reactividad del lecho vascular pulmonar frente a iloprost inhalado fueron evaluadas ecocardiográficamente en los pacientes con HP.

Resultados: Los pacientes con HP presentaron FDD disminuida versus los controles ( $2,8 \pm 0,6$ vs $10,7 \% \pm 0,6$, $\mathrm{p}<0,01)$. Niveles de MDA y eXO aumentados $(0,61 \pm$ 0,17 vs $0,34 \pm 0,15 \mu \mathrm{M}, \mathrm{p}<0,01$ y $0,039 \pm 0,005$ vs 0,034 $\pm 0,004 \mathrm{U} / \mathrm{mLl}, \mathrm{p}=0,02$ respectivamente) y actividad de eSOD disminuida $(235,55 \pm 23$ vs $461,41 \pm 33 \mathrm{ABC}$, $\mathrm{p}<0,01)$. Iloprost mejora significativamente el gasto cardíaco derecho y disminuye la resistencia vascular pulmonar en los pacientes con HP y este cambio se correlaciona con la actividad de eSOD (Rho: $0,61, \mathrm{p}<0,01$ ) y FDD (Rho: 0,63,p=0,01).

Conclusiones: Pacientes con HP presentan parámetros de estrés oxidativo elevados y disfunción endotelial periférica. La respuesta hemodinámica frente al uso de Iloprost se correlaciona con estos parámetros sugiriendo un rol en la HP cuyo valor clínico deberá ser evaluado. 
Increased oxidative stress are associated with peripheral endothelial dysfunction and diminished vascular response to vasodilators in pulmonary hypertensive patients

Background: Pulmonary Arterial Hypertension (PAH) is characterized by endothelial dysfunction and vascular remodeling. Several lines of experimental evidence indicate that oxidative stress plays an important role in the pathogenesis of PAH. The role of oxidative stress and its relation with peripheral endothelial function and pulmonary vascular response to vasodilators remains unknown.

Aim: To evaluate whether systemic oxidative stress and endothelial dysfunction markers are associated with the response of the pulmonary vascular bed to inhaled vasodilators in PAH patients.

Methods: Cross-sectional study. Fourteen patients with PAH and 14 age and gender-matched controls were included. Systemic oxidative stress was assessed through plasma malondialdehyde (MDA), xanthine oxidase (eXO) levels and endothelial-bound superoxide dismutase (eSOD) activity. Brachial artery endothelial-dependent flow-mediated vasodilation (FDD) was used to evaluate endothelial function. Right ventricular function and pulmonary vascular bed reactivity to inhaled vaso-

\section{Introducción:}

La Hipertensión Arterial Pulmonar (HP) es una patología crónica y progresiva que se caracteriza por un aumento de la resistencia vascular pulmonar debido al remodelado y oclusión de pequeñas arteriolas de la vasculatura pulmonar. En ausencia de tratamiento lleva a la hipertrofia y dilatación ventricular derecha (VD) produciéndose falla VD y muerte ${ }^{1}$.

El cateterismo cardíaco continúa siendo el "gold standard" para el diagnóstico de la HP, evaluando la severidad de la enfermedad, determinando el pronóstico y la respuesta a la terapia ${ }^{2}$. Aunque la ecocardiografía ha sido utilizada como una alternativa no invasiva para estos fines, tiene algunas limitaciones y existe evidencia contradictoria sobre la fuerza de la correlación entre la presión sistólica del VD y la presión arterial pulmonar media (PAPm) evaluada por cateterismo derecho ${ }^{2,-5}$. Por otro lado, aunque la ecocardiografía se utiliza ampliamente en clínica para el manejo de pacientes con HP, no existe una prueba no invasiva adecuadamente validada para la estratificación de estos pacientes ${ }^{6,7}$. Recientes avances en la fisiopatología de la HP han mostrado alteraciones sistémicas que dilators was determined with echocardiography in PAH patients.

Results: Compared to controls, $\mathrm{PAH}$ patients showed impaired FDD $(2.8 \pm 0.6$ vs $10.7 \% \pm 0.6, \mathrm{p}<0.01)$, increased MDA and eXO levels $(0.61 \pm 0.17$ vs $0.34 \pm$ $0.15 \mu \mathrm{M}, \mathrm{p}<0.01$ and $0.039 \pm 0.005$ vs $0.034 \pm 0.004 \mathrm{U} /$ $\mathrm{mL}, \mathrm{p}=0.02$, respectively) and decreased eSOD activity $235.55 \pm 23$ vs $461.41 \pm 33$ AUC, $p<0.01)$. Iloprost significantly improved right cardiac output (RCO) and decreased pulmonary vascular resistance. The amount of change in RCO after iloprost inhalation correlated significantly with baseline eSOD activity and FDD ( Rho: $0.61, p<0.01$ and Rho: $0.63, \mathrm{p}=0.01$ respectively). Conclusions: PAH patients show increased oxidative stress and endothelial dysfunction markers. Response to inhaled iloprost is closely related with baseline endothelial function and oxidative stress parameters, suggesting an important role of these elements that requires further clinical evaluation.

Key words: pulmonary artery hypertension, oxidative stress, endothelial dysfunction.

podrían ser utilizadas como biomarcadores para evaluar la severidad y pronóstico de pacientes con $\mathrm{HP}^{8}$.

Evidencia acumulada muestra una alteración en la función endotelial del lecho vascular pulmonar como un elemento clave en la patogénesis de la $\mathrm{HP}^{9}$. El daño de la célula endotelial altera la producción de mediadores vasoactivos, disminuyendo la disponibilidad de óxido nítrico (NO) y prostaciclinas y aumentando endotelina-1, quimiokinas y tromboxano A $2{ }^{9}$, provocando un aumento en el tono y remodelado del lecho vascular pulmonar, alteraciones características de la HP ${ }^{9}$. Interesantemente, al comparar con controles los pacientes con HP presentan alteración de la función endotelial periférica, lo que se correlaciona con la respuesta vascular pulmonar con el uso de vasodilatadores ${ }^{8}$.

Otro elemento importante que participa en la fisiopatología de la HP es el estrés oxidativo (revisado en ${ }^{10-11}$ ). Especies oxidantes (por ejemplo: aniones superóxido, radicales hidroxilos y peroxilos, como también H2O2) son bioproductos del metabolismo humano, los cuales en situaciones normales son eficientemente detoxificados por su interacción con "scavengers" endógenos y enzimas antioxidantes, por ejemplo: catalasa, glutatión peroxidasa y supe- 
róxido dismutasa ligada a endotelio (eSOD). Cualquier alteración en el balance a favor de las especies oxidantes resulta en un incremento del estrés oxidativo, llevando a daño celular directo por oxidación de membranas lipídicas, proteínas y DNA ${ }^{12}$. En enfermedades cardiovasculares el estrés oxidativo ha sido implicado en la patogénesis de condicione, tales como insuficiencia cardíaca, hipertrofia ventricular, HP e hipertensión sistémica ${ }^{10,13,14}$. Ambos, el estrés oxidativo y la disfunción endotelial son procesos ligados que llevan al remodelado vascular y al progreso de la enfermedad.

Nuestra hipótesis es que marcadores de estrés oxidativo sistémico y métodos de evaluación de la función endotelial periférica están relacionados entre si y con la respuesta vascular pulmonar frente al uso de vasodilatadores en pacientes con HP y podrían servir como biomarcadores para identificar que pacientes se beneficiarían más con la terapia. Este estudio piloto tiene como objetivo evaluar si los avances recientes en la fisiopatología y nuevas terapias en la HP se pueden trasladar a herramientas de utilidad clínica para la evaluación de los pacientes.

\section{Pacientes y métodos:}

Estudio transversal. Se incluyeron pacientes con HP, clase 1 de la clasificación de Venecia, evaluados en nuestro Hospital entre diciembre 2007 y mayo 2009. Los criterios de inclusión fueron: mayor de 18 años, diagnóstico de HP clase 1 con $\mathrm{PAPm} \geq 30 \mathrm{mmHg}$ en reposo y presión de capilar pulmonar $<15 \mathrm{mmHg}$ al momento del cateterismo derecho, tratamiento estable en los últimos tres meses, sin evidencia de enfermedad tromboembólica por estudio de angioTAC. Criterios de exclusión: embarazo, uso crónico de iloprost, condición médica concomitante que altere parámetros de estrés oxidativo, insuficiencia renal (creatinina $>2 \mathrm{mg} / \mathrm{dL}$ ), enfermedad autoimmune, neoplasia, enfermedad hepática o pulmonar avanzada, inflamación aguda o crónica. Para comparaciones basales se incluyó un grupo control de personas sanas, no fumadores pareados por edad y sexo. Todos los sujetos del estudio firmaron un consentimiento aprobado por un comité de ética local.

Todos los pacientes fueron sometidos a una prueba de vasoreactividad utilizando iloprost inhalatorio $(20 \mu \mathrm{g} / 4.5$ $\mathrm{mL}$ en solución salina, Ventavis ${ }^{\circledR}$, Bayer-Schering Pharma, Alemania) aplicado mediante un nebulizador jet por 10 minutos $^{15}$

\section{Evaluación de función endotelial periférica}

Todos los estudios de función endotelial se realizaron an- tes de las 09 am. Los pacientes permanecieron en ayuno y en reposo en una habitación tranquila por 30 minutos previo al examen. Un transductor lineal de 7,5 $\mathrm{MHz}$ fue utilizado para evaluar en forma longitudinal la arteria braquial 3-5 centímetros sobre el pliegue del codo. La vasodilatación dependiente de endotelio mediada por flujo (FDD) fue evaluada inflando un manguito de presión a $300 \mathrm{mmHg}$ en el antebrazo. Luego de 5 minutos de oclusión del flujo arterial el manguito fue desinflado. Se midió el diámetro de la arteria braquial pre y post oclusión. Un aumento del diámetro de la arteria braquial menor a $8 \%$ fue considerada como evidencia de disfunción endotelial ${ }^{16}$. La vasodilatación independiente de endotelio fue evaluada 10 minutos luego del desinflado del manguito, midiendo el diámetro de la arteria braquial basalmente y a los 3 minutos post administración de 1,25 mg de dinitrato de isosorbide en spray sublingual (Schwarz Pharma AG, Swiss).

\section{Evaluación de la respuesta vascular pulmonar}

Un ecocardiograma transtorácico fue realizado en todos los sujetos (Philips IE-33) basalmente, a los 15 y 45 minutos posterior a la inhalación de iloprost. El examen fue realizado por un operador experto y ciego. Se midió y calculó: presión arterial pulmonar media (PAPm), resistencia vascular pulmonar (RVP), desplazamiento longitudinal del anillo tricuspídeo lateral en sístole (TAPSE), doppler tisular a nivel de anillo tricupídeo y el gasto cardíaco derecho $(\mathrm{GC})^{17}$.

\section{Evaluación de estrés oxidativo}

Basalmente en todos los sujetos se obtuvo una muestra de sangre venosa de una vena antecubital del brazo no dominante. Posteriomente se administró un bolo de heparina (5000 UI) en la arteria braquial del mismo brazo y muestras de la vena antecubital fueron tomadas a intervalos predefinidos ( ${ }^{1,3,5,7}$ y 10 minutos post inyección de heparina). La actividad de eSOD fue obtenida mediante el cálculo del área bajo la curva $(\mathrm{ABC})$ de la actividad plasmática de SOD expresada en unidades por $\mathrm{mL}$ de plasma como fue descrito previamente ${ }^{18}$.

La actividad de xantino oxidasa endotelial (eXO) fue calculada como la diferencia entre la actividad basal de XO plasmática y 5 minutos después de una inyección intraarterial de heparina ${ }^{19,20} \mathrm{y}$ fue expresada como unidades de absorbancia (AU) por $\mathrm{mL}$ de plasma por minuto.

Los niveles plasmáticos de malondialdehído (MDA), un marcador de peroxidación lipídica, fueron determinados 


\begin{tabular}{llll|}
\hline \multicolumn{4}{|c|}{ Tabla 1: Características demográficas y ecocardiográficas. } \\
\hline & Pacientes $(\mathrm{n}=14)$ & Controles $(\mathrm{n}=14)$ & $\mathrm{p}$ \\
\hline Edad (a os) & $38 \pm 7$ & $31 \pm 7$ & $\mathrm{NS}$ \\
Mujeres (\%) & 61 & 64 & $\mathrm{NS}$ \\
PSAP (mmHG) & $78,5 \pm 9$ & $24 \pm 4$ & $<0,01$ \\
PMAP (mmHG) & $50,5 \pm 5$ & $12 \pm 3$ & $<0,01$ \\
TAPSE (mm) & $15 \pm 3$ & $22 \pm 4$ & 0,03 \\
Tiempo aceleraci $\mathrm{n}$ ITV TSVD (mseg) & $68 \pm 10$ & $119 \pm 10$ & $<0,01$ \\
Onda sist lica ATL (cm/seg) & $10 \pm 2$ & $15 \pm 3$ & 0,02 \\
Diámetro diastólico VD (mm) & $44 \pm 6$ & $21 \pm 4$ & $<0,01$
\end{tabular}

Abreviaciones: PSAP: presión sistólica de arteria pulmonar; PMAP: presión media arteria pulmonar; TAPSE: excursión sistólica anillo tricuspídeo lateral; ITV TSVD: integral tiempo-volumen tracto salida ventrículo derecho; ATL: anillo tricuspídeo lateral, doppler tisular; VD: ventrículo derecho.

mediante la evaluación del contenido de substancias reactivas al ácido tiobarbitúrico como fue descrito previamente ${ }^{13}$.

\section{Análisis estadístico}

Las diferencias basales entre los pacientes con HP y el grupo control se evaluaron con el test de chi-cuadrado o Mann-Whitney U test según correspondía. El cambio en los parámetros hemodinámicos post inhalación de iloprost se evaluaron mediante Wilcoxon Rank sum test. Las correlaciones entre la respuesta hemodinámica al iloprost inhalado, la FDD y los parámetros de estrés oxidativo fueron evaluados con el método de Spearman. Las variables categóricas se expresaron como porcentajes y las continuas como promedios \pm desviación estándar. Un valor $\mathrm{p}$ $<0,05$, dos colas fue considerado significativo.

\section{Resultados:}

Se incluyeron 14 pacientes con HP y 14 controles pareados por edad y sexo. La edad promedio de los pacientes con HP fue $38 \pm 7$ años y $31 \pm 7$ los controles, 9 pacientes con HP eran mujeres (61\%). La terapia de los pacientes era: ambrisentan $100 \%$, sildenafil $58 \%$ y cumarínicos un $52 \%$. Ninguno de los pacientes utilizaba oxígeno domiciliario. En los pacientes con HP el test de caminata 6 minutos fue $394 \pm 55$ metros. Las características demográficas y ecocardiográficas basales se muestran en la tabla 1 .

\section{Función endotelial periférica y parámetros de estrés oxidativo en los pacientes con HP.}

Basalmente los pacientes con HP presentaban FDD disminuida respecto del grupo control, Figura 1.Por otro lado los parámetros de estrés oxidativo fueron consistentes con un estado pro-oxidante asociado a mayor peroxidación lipídica, mayor de actividad de eXO y menor actividad de eSOD respecto al grupo control sano. Los parámetros de estrés oxidativo se detallan en la tabla 2.

\section{Efectos del iloprost en el gasto cardíaco y resistencia vascular pulmonar en pacientes con HP.}

En los pacientes con HPel iloprost inhalatorio produce un aumento significativo del GC derecho y una disminución de la RVP, Figura 2 y 3 . Se observó una correlación positiva significativa entre FDD, mejoría en el GC y actividad

\begin{tabular}{|llll|}
\multicolumn{4}{c}{ Tabla 2: Par metros de estr s oxidativo } \\
\hline \\
\hline HP & Controles & p \\
\hline XO $(\mathrm{U} / \mathrm{mL})$ & $0,039 \pm 0,005$ & $0,034 \pm 0,004$ & 0,02 \\
MDA $(\mu \mathrm{M})$ & $0,61 \pm 0,17$ & $0,34 \pm 0,15$ & $<0,001$ \\
eSOD (ABC) & $235,55 \pm 23$ & $461,41 \pm 33$ & $<0,01$ \\
\hline
\end{tabular}

Abreviaciones: XO: xantino oxidasa; MDA: malondialdehído; eSOD: superóxido dismutasa ligada a endotelio; $A B C$ : área bajo la curva. 

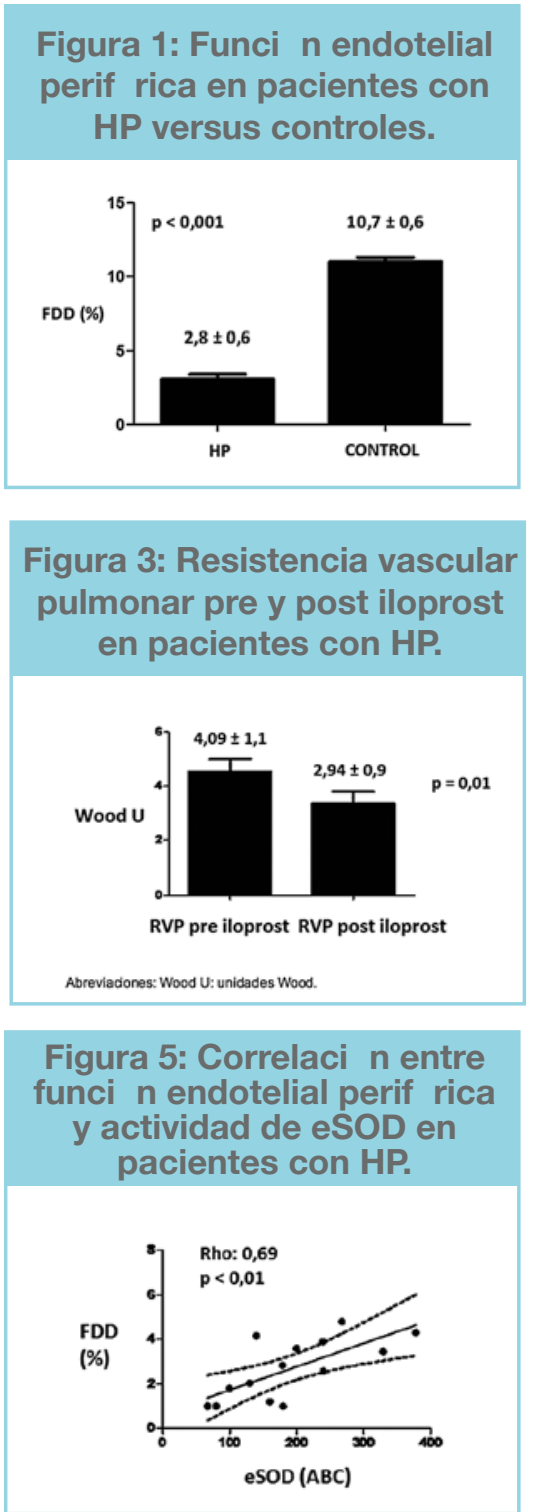

de eSOD, Figura 4, 5 y 6. Por otro lado, los pacientes con HP presentaron una correlación negativa significativa entre la actividad de eXO y la mejoría en el GC post iloprost $(\mathrm{Rho}=-0,65 ; \mathrm{p}=0,01)$ y entre $\mathrm{eXO}$ y FDD $(\mathrm{Rho}=-0,42$; $\mathrm{p}=0,03)$. Las correlaciones entre MDA y mejoría del GC y MDA con FDD no fueron significativas, $(\mathrm{Rho}=-0,21$; $\mathrm{p}=0,5)$ y $(\mathrm{Rho}=-0,34 ; \mathrm{p}=0,2)$ respectivamente.

\section{Discusión:}

Los principales hallazgos del presente trabajo fueron que pacientes con HP presentan: a) disfunción endotelial periférica, aumento en el estrés oxidativo caracterizado por aumento en los niveles de MDA y cambios en la actividad de enzimas endoteliales ligadas a la producción (eXO) y catabolismo (eSOD) de especies reac-
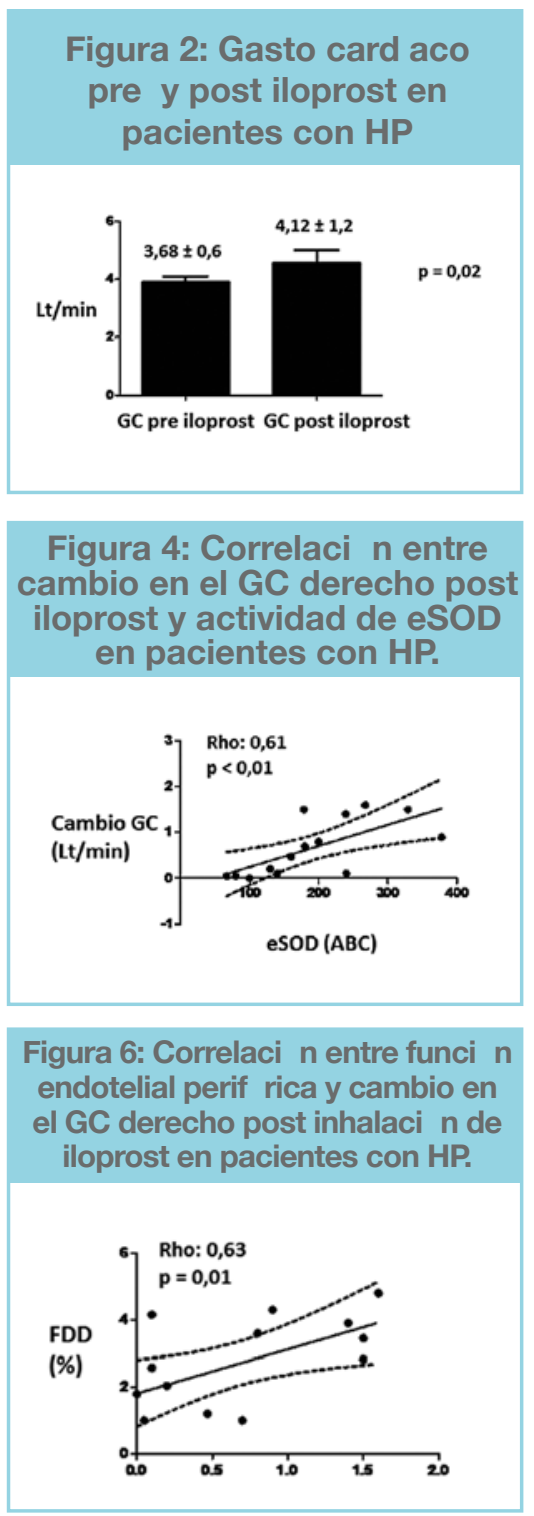

tivas de oxígenos (ERO). b) la actividad de eSOD se correlaciona con la FDD y la mejoría en el GC derecho con el uso de iloprost en pacientes con HP.

En el presente estudio se corrobora el hallazgo de disfunción endotelial periférica en pacientes con HP y que la respuesta vascular pulmonar frente al uso de vasodilatadores está relacionada a esta disfunción ${ }^{8}$. El endotelio pulmonar normal es heterogéneo y difiere en forma significativa del endotelio de la circulación sistémica en su ultraestructura y función ${ }^{21}$. La principal función del endotelio pulmonar es la mantención del tono vascular, homeostasis, tráfico de leucocitos, producción de factores de crecimiento, entre otros ${ }^{22}$. El endotelio libera una serie de mediadores vasoactivos que regulan las propiedades físicas y bioquímicas de la vasculatura pulmonar afectando su contractilidad y proliferación 
celular $^{23}$. Por tanto, la disfunción endotelial parece jugar un rol integral en mediar los cambios estructurales en la vasculatura pulmonar de los pacientes con HP. Los mecanismos responsables de la disfunción endotelial en la hipertensión pulmonar no están del todo aclarados y un aumento del estrés oxidativo, con un compromiso de la actividad antioxidante de eSOD parece explicar, al menos en parte, esta disfunción observada en estos pacientes.

Diferentes estudios han implicado al estrés oxidativo en el desarrollo de disfunción endotelial en la patogénesis de las enfermedades cardiovasculares ${ }^{10-11}$. El aumento del estrés oxidativo es producido por un desbalance entre los mecanismos de generación de ERO y los sistemas antioxidantes enzimáticos y no enzimá$\operatorname{ticos}^{12}$. El estrés oxidativo deteriora la función cardiovascular al modificar membranas lipídicas, DNA y proteínas causando disfunción y muerte celular ${ }^{12}$. El anión superóxido, potente inactivador de óxido nítrico, contribuye a la disfunción endotelial y a la producción de peroxinitritos, especie más potente que el anión superóxido ${ }^{11}$. Las ERO están involucradas en la degradación de la matriz extracelular particularmente por activación de metaloproteasas en la vasculatura pulmonar ${ }^{10}$. Los isoprostanos se forman cuando las ERO reaccionan con membranas lipídicas insaturadas $^{24}$ y se acumulan en forma significativa en estados de aumento del estrés oxidativo y se han ocupado como marcadores de severidad de la enfermedad. Más aún, nueva evidencia sugiere un rol importante de los isoprostanos en la patología vascular pulmonar, teniendo éstos un potente efecto vasoconstrictor a través de su acción sobre el endotelio para la liberación de endotelina, un potente vasoconstrictor ${ }^{25}$. Por otro lado, niveles elevados de isoprostanos se han encontrado en modelos experimentales de $\mathrm{HP}^{11}$.

Las fuentes de ERO incluyen la infiltración del tejido por células inflamatorias, las mitocondrias, $\mathrm{XO}$ y NADPH oxidasa ${ }^{12}$. A nivel vascular eSOD y eXO son los principales mecanismos antioxidantes y de generación de anión superóxido respectivamente ${ }^{19,20}$. Ambas enzimas están ligadas al endotelio periférico y pulmonar, y son liberadas ante bolos de heparina ${ }^{19}$, 20. En pacientes con insuficiencia cardíaca crónica nuestro grupo describió la presencia de disfunción endotelial periférica y su asociación con niveles aumentados de MDA circulante, y la correlación entre actividad disminuida de eSOD y el grado de disfunción endotelial post terapia con atorvastatina en los mismos pacientes ${ }^{18}$. De manera que, el estrés oxidativo parece ser un elemento central en los mecanismos fisiopatológicos de la disfunción endotelial observada en los pacientes con insufuciencia cardíaca y, por los hallazgos del presente estudio, en los pacientes con HP. Niveles aumentados de estrés oxidativo se observan en situaciones de disfunción ventricular ${ }^{14}$, sin embargo, los pacientes de nuestro estudio no tenían evidencia clínica ni ecocardiográfica (según TAPSE) de disfunción ventricular derecha.

En general, el tratamiento para la HP ha sido básicamente de soporte y ha incluído: oxígeno, calcio antagonistas, diuréticos y evitar la actividad física ${ }^{27}$. Recientemente, la incorporación de vasodilatadores pulmonares, como los análogos de prostaciclina, ha mejorado la calidad de vida y pronóstico de estos pacientes. Este grupo de drogas han mostrado disminución en la proliferación de células musculares lisas de arterias pulmonares y de la agregación plaquetaria ${ }^{28}$. Cabe destacar, que los análogos de prostaciclina han mostrado reducción en los niveles de estrés oxidativo y mejoría en la función cardíaca en adición a

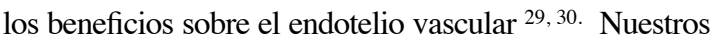
resultados concuerdan con estos conceptos, mostrando mejoría en el GC derecho con el uso de vasodilatadores asociado a un estado menos prooxidante. Sin embargo, los niveles de MDA no se correlacionaron con esta respuesta, indicando que probablemente los sistemas más comprometidos en estos pacientes están relacionados con el catabolismo de las ERO por lo antioxidantes y no tanto la generación éstos.

En conjunto estos resultados sugieren un rol del estrés oxidativo en el desarrollo de hipertensión pulmonar, remodelado vascular pulmonar y disfunción endotelial y podrían constituir un objetivo terapéutico que requiere ser evaluado. 


\section{Referencias:}

1. D'alonzo GE, Barst RJ, Ayres SM, Bergofsky EH, Brundage BH, Detre KM, et al. Survival in patients with primary pulmonary hypertension: results from a national prospective registry. Ann Intern Med 1991; 115: 343-9

2. Champion HC, Michelakis ED, Hassoun PM. Comprehensive invasive and noninvasive approach to the right ventricle-pulmonary circulation unit: state of the art and clinical and research implications. Circulation. 2009; 120: 992-1007.

3. Berger M, Haimowitz A, Van Tosh A, Berdoff RL, Goldberg E. Quantitativ assessment of pulmonary hypertension in patients with tricuspid regurgitation using continuous wave Doppler ultrasound. J Am Coll Cardiol. 1985; 6: 35965.

4. Currie PJ, Seward JB, Chan KL, Fyfe DA, Hagler DJ, Mair $\mathrm{DD}$, et al. Continuous wave Doppler determination of right ventricular pressure: a simultaneous Doppler-catheterization study in 127 patients. J Am Coll Cardiol. 1985; 6: 7506

5. Devaraj A, Wells AU, Meister MG, Corte TJ, Wort SJ, Hansell DM. Detection of pulmonary hypertension with multidetector CT and echocardiography alone and in combination. Radiology. 2010; 254: 609-16

6. Tan RT, Kuzo R, Goodman LR, Siegel R, Haasler GB, Presberg KW.. Utility of CT scan evaluation for predicting pulmonary hypertension in patients with paren parenchymal lung disease. Medical College of Wisconsin Lung Transplant Group. Chest 1998; 113: 1250-6.

7. Perez-Enguix D, Morales P, Tomas JM, Vera F, Lloret RM. Computed tomographic screening of pulmonary arterial hypertension in candidates for lung transplantation. Transplant Proc 2007; 39: 2405-8.

8. Wolff B, Lodziewski S, Bollmann T, Opitz Cf, Ewert R. Impaired peripheral endothelial function in severe idiopathic pulmonary hypertension correlates with the pulmonary vascular response to inhaled iloprost. Am Heart J. 2007; 153: 1088e1-1088e7.

9. Dinh-Xuan AT. Endothelial modulation of pulmonary vascular tone. Eur Respir J. 1992; 5: 757-62.

10. Bowers R, Cool C, Murphy RC, Tuder RM, Hopken MW, Flores SC, et al. Oxidative stress in severe pulmonary hypertension. Am J Respir Crit Care Med 2004; 169: 764-9.

11. Crosswhite P, Sun Z. Nitric oxide, oxidative stress and inflammation in pulmonary arterial hypertension. J Hypertens. 2010; 28: 201-12.

12. Cadenas E, Sies H. Oxidative stress: excited oxygen species and enzyme activity. Adv Enzyme Regul. 1985; 23:
217-37.

13. Castro PF, Díaz-Araya G, Nettle D, Corbalán R, Pérez O, Nazzal C, et al. Effects of early decrease in oxidative stress after medical therapy in patients with class IV congestive heart failure. Am J Cardiol. 2002; 89: 236-9.

14. Castro PF, Greig D, Pérez O, Moraga F, Chiong M, DíazAraya G, et al. Relation between oxidative stress, catecholamines, and impaired chronotropic response to exercise in patients with chronic heart failure secondary to ischemic or idiopathic dilated cardiomyopathy. Am J Cardiol. 2003; 92: $215-8$

15. Jing ZC, Jiang X, Han ZY, Xu XQ, Wang Y, Wu Y, et al. Iloprost for pulmonary vasodilator testing in idiopathic pulmonary arterial hypertension. Eur Respir J. 2009.

16. Celermajer DS. Testing endothelial function using ultrasound. J Cardiovasc Pharmacol 1998; 32: S29-32.

17. Selimovic N, Rundqvist B, Bergh Ch, Andersson B, Petersson S, Johansson L, et al. Assessment of pulmonary vascular resistance by Doppler echocardiography in patients with pulmonary arterial hypertension. J Heart Lung Transplant. 2007; 26: 927-34.

18. Castro PF, Miranda R, Verdejo HE, Greig D, Gabrielli LA, Alcaino $\mathrm{H}$, et al. Pleiotropic effects of atorvastatin in heart failure: role in oxidative stress, inflammation, endothelial function, and exercise capacity. J Heart Lung Transplant. 2008; 27: 435-41

19. Prajda N, Weber G. Malignant transformation-linked imbalance: decreased xanthine oxidase activity in hepatomas. FEBS Lett. 1975; 59: 245-249.

20. Landmesser U, Spiekermann S, Dikalov S, Tatge H, Wilke $\mathrm{R}, \mathrm{Kohler} \mathrm{C}$, et al. Vascular oxidative stress and endothelial dysfunction in patients with chronic heart failure: role of xanthine-oxidase and extracellular superoxide dismutase. Circulation. 2002; 106: 3073-3078.

21. Tuder RM, Cool Cd, Yeager M, Taraseviciene-Stewart L, Bull TM, Voelkel NF. The pathobiology of pulmonary hypertension. Endothelium. Clin Chest Med. 2001; 22:405 18.

22. Morrell NW, Adnot S, Archer SL, Dupuis J, Jones PL, Maclean MR, et al. Cellular and molecular basis of pulmonary arterial hypertension. J Am Coll Cardiol. 2009; 54: S20 31.

23. Farber HW, Loscalzo J. Pulmonary arterial hypertension. N Engl J Med. 2004; 351: 1655-65.

24. Janssen LJ. Isoprostanes and lung vascular pathology. Am J Respir Cell Mol Biol 2008; 39: 383-9

25. Yi SL, Kantores C, Belcastro R, Cabacungan J, Tanswell Ak, Jankov Rp. 8 Isoprostane-induced endothelin-1 production by infant rat pulmonary artery smooth muscle cells is mediated by Rho-kinase. Free Radic Biol Med 2006; 
Niveles aumentados de estr s oxidativo se asocian a disfunci $n$ endotelial perif rica y respuesta vascular pulmonar disminuida...

41:942-9.

26. Pan JQ, Li JC, Tan X, Sun WD, Wang JY, Wang XL. The injury effect of oxygen free radicals in vitro on cultured pulmonary artery endothelial cells from broilers. Res Vet Sci. 2007; 82: 382-7.

27. Hrometz SL, Shields KM. Role of ambrisentan in the management of pulmonary hypertension. Ann Pharmacother 2008; 42:1653-1659

28. Yildiz P. Molecular mechanisms of pulmonary hyperten- sion. Clin Chim Acta 2009; 403: 9-16.

29. Ferrari R, Cargnoni A, Curello S, Boffa GM, Ceconi C. Effects of iloprost (ZK 36374) on glutathione status during ischaemia and reperfusion of rabbit isolated hearts. $\mathrm{Br} \mathrm{J}$ Pharmacol 1989; 98: 678-684;

30. Shinmura K, Tamaki K, Sato T, Ishida H, Bolli R. Prostacyclin attenuates oxidative damage of myocytes by opening mitochondrial ATP-sensitive $\mathrm{K}+$ channels via the EP3 receptor. Am J Physiol 2005; 288: H2093-210. 